\title{
CONHECIMENTO EAPLICAÇÃO DO DIREITO DO ACOMPANHANTE NA GESTAÇÃO E PARTO
}

Eliene Correia Pereira dos Santos¹, Mônica Reis Lima', Leila Luiza Conceição², Catiane Souza Tavares ${ }^{3}$, Alzira Maria d'Ávila Nery Guimarães ${ }^{3}$

Objetivo: identificar o cumprimento da lei 11.108/2005, que assegura às mulheres o direito do acompanhante, de sua livre escolha, durante o período de trabalho de parto, parto e pós-parto imediato. Metodologia: pesquisa de corte transversal, desenvolvida no período de abril a maio de 2015, em duas maternidades filantrópicas, conveniadas ao Sistema Único de Saúde do estado de Sergipe, uma na capital e outra no interior do estado. A amostra constou de 160 gestantes e puérperas. Resultados: verificouse que 92 (57,5\%) das entrevistadas não conheciam a lei referida, e 106 (66,2\%) não dispuseram da presença do acompanhante em todo o processo de parturição. Observou-se, ainda, que apenas 64 (41,8\%) das puérperas tiveram acompanhante no pósparto imediato. Conclusão: os resultados reforçam a importância de novas discussões relativas a esse direito constituído.

Descritores: Parto humanizado; saúde da mulher; parto.

\section{KNOWLEDGE AND APPLICATION OF THE RIGHT OF THE ACCOMPANYING IN PREGNANCY AND BIRTH}

Objective: to identify the compliance with the Law 11,108 / 2005, which grants women the right companion of their choice during labor and delivery period, delivery and immediate postpartum . Methodology: cross-sectional study, carried out from April to May 2015 in two charitable maternity hospitals to the Unified Health System in the state of Sergipe, one in the capital and another in the state. The sample consisted of 160 pregnant and postpartum women. Results: It was found that 92 (57.5 $\%$ ) of respondents did not know the Law, and 106 ( $66.2 \%$ ) were not provided of the partner's presence throughout the parturition process. It was also observed that only 64 (41.8\%) of the mothers were accompanying the immediate post -partum . Conclusion: the results reinforce the importance of further discussions regarding this right constituted, to be developed in order to fulfill what determines the Law 11,108 / 2005 , an achievement still not effectively achieved.

Descriptors: Humanizing delivery; Health of Women; parturition.

\section{CONHECIMENTO Y APLICACIÓN DO DIREITO DO ACOMPANHANTE NA GESTAÇÃO E PARTO}

Objetivo: identificar el cumplimiento de la Ley 11.108 / 2005, que otorga a la mujer el compañero derecho de su elección durante el parto y el período de entrega, la entrega y el posparto inmediato. Metodología: Estudio transversal, realizado entre abril y mayo de 2015, de dos hospitales de maternidad de caridad para el Sistema Único de Salud en el estado de Sergipe, uno en la capital y otro en el estado. La muestra consistió en 160 mujeres embarazadas y posparto. Resultados: se encontró que 92 (57,5\%) de los encuestados no conocen la ley, y 106 (66,2\%) no se proporcionaron de la presencia de la pareja durante todo el proceso del parto. También se observó que sólo 64 (41,8\%) de las madres acompañaban el inmediatamente posterior al parto. Conclusión: los resultados refuerzan la importancia de los nuevos debates en relación con este derecho constituido, para ser desarrollado con el fin de cumplir con lo que determina la Ley 11.108 / 2005, un logro todavía no ha alcanzado efectivamente.

Descriptores: Humanización del parto; Salud de la Mujer; parto

${ }^{1}$ Estudante do curso de Enfermagem da Universidade Federal de Sergipe,UFS

${ }^{2}$ Enfermeira. Mestranda da UFS.

${ }^{3}$ Enfermeira. Doutora em Enfermagem. Professora da UFS. E-mail: alziranery@uol.com.br 


\section{INTRODUÇÃO}

Ao longo dos anos, diferentes significados foram atribuídos à gestação e ao parto.(1) Com novas tecnologias desenvolvidas na medicina, a mulher deixa de ter autonomia e privacidade, sendo separada do âmbito familiar e submetida às regras institucionais e práticas intervencionistas, sem a devida elucidação e aquiescência da parturiente. Dessa forma, o parto se tornou, em alguns casos, perda de princípios e intenso sofrimento físico. (2)

Visando que o parto normal possa ser uma escolha informada e segura para a mulher, o Ministério da Saúde vem estimulando a implementação de políticas incentivadoras do parto normal humanizado, a exemplo, a Política Nacional de Humanização do Parto e do Nascimento (PNHPN), a Estratégia Rede Cegonha, dentre outras. ${ }^{(3)}$

Ainda, para contribuir com o parto humanizado, foi aprovada e sancionada, em de 07 de abril de 2005, a Lei $\mathrm{n}$ ㅇ 11.108, nominada "Lei Acompanhante", respaldando os integrantes do Sistema Único de Saúde (SUS) a permitir, à parturiente, um acompanhante de sua preferência, durante todo o processo de trabalho de parto, parto e pós-parto.(4)

Embora haja respaldo legal, um contingente elevado de gestantes não tem seu direito garantido em várias maternidades brasileiras. Frequentemente, as parturientes são privadas da presença de um acompanhante, de sua escolha, para thes dar apoio no processo do nascimento, em decorrência das práticas assistenciais adotadas nos serviços de saúde. $\mathrm{O}$ abandono de práticas inadequadas potencialmente danosas, no parto, depende tanto da motivação e compromisso dos profissionais quanto da estrutura de muitas maternidades. ${ }^{(5)}$

Para conhecer a realidade de maternidades do estado de Sergipe em cumprimento da lei 11.108/2005, este estudo tem como objetivo identificar o conhecimento e a aplicação da lei que garante o acompanhante na atenção à gestação e ao parto.

\section{METODOLOGIA}

Estudo quantitativo, descritivo, de corte transversal, realizado com gestantes, parturientes e puérperas em duas maternidades filantrópicas do estado de Sergipe, uma localizada na capital e outra no interior do estado, conveniadas ao SUS.

Ambas são instituições beneficentes e sem fins lucrativos. A maternidade da capital (A), unidade de referência para baixo risco obstétrico, eventualmente atende puérperas de alto risco, realizando aproximadamente 1200 partos mensais. ${ }^{(6)} \mathrm{A}$ maternidade do interior do estado (B), atende gestantes de risco habitual, urgências e emergências obstétricas da região, com média de 200 partos $/$ mês $^{(7)}$.

A amostra do estudo foi intencional, constituída por mulheres que pariram nas duas maternidades de abril a maio de 2015, nos dias de coleta de dados: segundas e quartas-feiras, no período matutino, e às sextas, no período vespertino, totalizando 160 gestantes/puérperas, 80 para cada maternidade.

A coleta de dados ocorreu mediante formulário testado, validado, contendo 32 perguntas fechadas, abordando as seguintes variáveis: características sóciodemográficas, da saúde reprodutiva, participação e importância do acompanhante durante a gestação, prénatal e parto. Como critério de inclusão, as gestantes, parturientes ou puérperas teriam que residir no estado de Sergipe, parirem nas maternidades acima referidas, nos dias eleitos para a coleta de dados e concordarem em participar do estudo.

Utilizou-se o Programa TabWin versão 3.4 .2 para tabulação dos dados, realizando-se a análise por estatística descritiva, apresentadas em frequências absolutas e relativas. O estudo foi aprovado pelo Comitê de ética em pesquisa, Campus da Saúde-Universidade Federal de Sergipe, com protocolo de número 42784515.5.0000.5546

\section{RESULTADOS}

A maioria das entrevistadas, 108 (67,5\%), encontrava-se na faixa etária entre 20 a 35 anos, viviam com companheiro, 147 (91,8\%), e declararam-se não brancas 92 (57,5\%) e não exercer atividades remuneradas, 102 (63,7\%), havendo semelhanças nos dois grupos pesquisados. As mulheres procedentes da maternidade localizada na capital (A) referiram possuir mais anos de estudo e maior renda familiar.

Nos aspectos relativos à saúde reprodutiva, as mulheres da maternidade $\mathrm{B}$ tinham maior paridade, (> 3 filhos), que as 
da A, 12 (15,0\%) e 6 (7,5\%) respectivamente. Quando analisada a idade gestacional, evidencia-se que 9 (11,2\%) das crianças eram prematuras, e 120 (75,0\%) fizeram mais de 6 consultas durante o pré-natal. Na categoria internação, 160 (100\%) das mulheres afirmaram ter como plano de saúde o SUS. Um contingente de 35 (43,7\%) das mulheres da maternidade A informou não ter tido participação do acompanhante no pré-natal, enquanto na outra maternidade, foi verificado um percentual maior, 46 (57,5\%), de mulheres que não dispuseram de um acompanhante durante as consultas na gestação.

Tabela 1 - Aspectos relativos ao conhecimento da "lei do acompanhante" das participantes do estudo realizado na Maternidade A (capital) e Maternidade B (interior) Sergipe, Brasil, 2015

\begin{tabular}{|c|c|c|c|}
\hline \multirow{3}{*}{ Variável (n) } & \multirow{3}{*}{$\begin{array}{l}A \\
N(\%)\end{array}$} & \multicolumn{2}{|c|}{ Maternidades } \\
\hline & & B & Total \\
\hline & & $N(\%)$ & Total (\%) \\
\hline \multicolumn{4}{|c|}{ Conhecimento da lei $11.108 / 2005$} \\
\hline Não & $45(56,2)$ & $47(58,8)$ & $92(57,5)$ \\
\hline Sim & $35(43,8)$ & $33(41,2)$ & $68(42,5)$ \\
\hline \multicolumn{4}{|c|}{$\begin{array}{l}\text { Foi informada durante o } \\
\text { pré-natal sobre a lei }\end{array}$} \\
\hline Não & $46(57,5)$ & $58(72,5)$ & $\begin{array}{l}104 \\
(65,0)\end{array}$ \\
\hline Sim & $34(42,5)$ & $22(27,5)$ & $56(35,0)$ \\
\hline \multicolumn{4}{|c|}{$\begin{array}{l}\text { Categoria do profissional } \\
\text { que informou }\end{array}$} \\
\hline Médico & $6(7,5)$ & $5(6,2)$ & $11(6,9)$ \\
\hline Enfermeiro & $18(22,5)$ & $9(11,3)$ & $27(16,9)$ \\
\hline Outro & $10(12,5)$ & $8(10,0)$ & $18(11,2)$ \\
\hline \multicolumn{4}{|l|}{$\begin{array}{l}\text { Maternidade informou } \\
\text { o direito de ter } \\
\text { acompanhante }\end{array}$} \\
\hline $\operatorname{Sim}$ & $50(62,5)$ & $14(17,5)$ & $64(40,0)$ \\
\hline Não & $30(37,5)$ & $66(82,5)$ & $96(60,0)$ \\
\hline \multicolumn{4}{|c|}{$\begin{array}{l}\text { Categoria do profissional } \\
\text { que informou }\end{array}$} \\
\hline Médico & $3(3,8)$ & $0(0,0)$ & $3(2,0)$ \\
\hline Enfermeiro & $17(21,3)$ & $4(5,0)$ & $21(13,0)$ \\
\hline Assistente Social & $1(1,2)$ & $4(5,0)$ & $5(3,0)$ \\
\hline Coordenação/ Outro & $29(36,2)$ & $6(7,5)$ & $35(22,0)$ \\
\hline
\end{tabular}

Fontes: Dados da pesquisa

$\mathrm{Na}$ Tabela 1, foi evidenciado que 92 (57,5\%) das entrevistadas não conheciam a referida lei; 46 (57,5\%) não receberam nenhuma informação na Maternidade A e 58 $(72,5 \%)$ na Maternidade B. As que informaram ter tido acesso ao conhecimento da lei, o adquiriram, com maior frequência, por meio do profissional enfermeiro 27 (16,9\%), apresentando percentuais diferentes entre as duas maternidades: 18 $(22,5 \%)$ para a Maternidade A e 09 (11,3\%) para a Maternidade B. Foi evidenciado que, por ocasião da internação, 50 (62,5\%) das mulheres assistidas na maternidade A foram informadas sobre esse direito, enquanto apenas 14 (17,5\%) da Maternidade $B$ tiveram essa informação, com maior frequência do informante para a categoria" coordenação da instituição".

Tabela 2 - Aspectos relativos ao processo da parturição das participantes do estudo realizado na Maternidade A (capital) e Maternidade B (interior) Sergipe, Brasil, 2015

\begin{tabular}{|c|c|c|c|}
\hline \multirow{3}{*}{ Variável (n) } & \multicolumn{3}{|c|}{ Maternidades } \\
\hline & A & B & Total \\
\hline & $N(\%)$ & $N(\%)$ & Total (\%) \\
\hline \multicolumn{4}{|c|}{$\begin{array}{l}\text { Maternidade permitiu o } \\
\text { acesso do acompanhante } \\
\text { em todo processo }\end{array}$} \\
\hline Não & $27(33,8)$ & $79(98,7)$ & $106(66,2)$ \\
\hline Sim & $53(66,2)$ & $1(1,3)$ & $54(33,8)$ \\
\hline \multicolumn{4}{|c|}{$\begin{array}{l}\text { Processo de parturição que } \\
\text { teve acompanhante }\end{array}$} \\
\hline Pré-parto & $1(1,3)$ & $2(2,5)$ & $3(2,0)$ \\
\hline Parto & $1(1,3)$ & $0(0,0)$ & $1(0,6)$ \\
\hline Pós-parto imediato & $16(20,0)$ & $51(63,7)$ & $67(41,8)$ \\
\hline Não se aplica & $9(11,2)$ & $26(32,5)$ & $35(21,8)$ \\
\hline \multicolumn{4}{|c|}{$\begin{array}{l}\text { Pessoa que acompanhou no } \\
\text { processo de parturição }\end{array}$} \\
\hline Mãe & $11(13,8)$ & $0(0,0)$ & $11(7,0)$ \\
\hline Companheiro & $21(26,2)$ & $0(0,0)$ & $21(13,2)$ \\
\hline Amigo (a) & $2(2,5)$ & $0(0,0)$ & $2(1,2)$ \\
\hline Outros (a) & $19(23,8)$ & $1(1,2)$ & $20(12,6)$ \\
\hline \multicolumn{4}{|c|}{$\begin{array}{l}\text { Privacidade durante todas } \\
\text { as etapas }\end{array}$} \\
\hline Sim & $54(67,5)$ & $34(42,5)$ & $88(55,0)$ \\
\hline Não & $26(32,5)$ & $46(57,5)$ & $72(45,0)$ \\
\hline \multicolumn{4}{|c|}{$\begin{array}{l}\text { Direito do acompanhante de } \\
\text { ter acomodação adequada } \\
\text { e receber as principais } \\
\text { refeições. }\end{array}$} \\
\hline Sim & $37(46,3)$ & $25(31,3)$ & $62(38,7)$ \\
\hline Não & $43(53,7)$ & $55(68,7)$ & $98(61,3)$ \\
\hline
\end{tabular}

Fontes: Dados da pesquisa

Analisando-se as características referentes ao processo de parturição (Tabela 2), o direito ao acompanhante foi superior entre as mulheres assistidas na maternidade A $(33,8 \%)$ 
quando comparadas às assistidas na maternidade B (1,3\%). Entre as etapas do processo de parturição, durante o parto, foi quando houve menor participação do acompanhante, ocorrendo somente na maternidade A, 1 (1,3\%). No pós-parto imediato, a participação do acompanhante foi maior entre as mulheres da maternidade B (63,7\%), quando comparada com as assistidas na maternidade A (20,0\%). Das mulheres que afirmaram ter tido acesso ao acompanhante, na Maternidade da capital (A), o companheiro foi o mais citado, 21 (26,2\%).

Em relação à privacidade (respeito as suas individualidades), durante a parturição, identificou-se que 54 (67,5\%) das participantes na maternidade A e 46 (57,5\%) na maternidade B informaram não terem sido contempladas. Quanto ao direito de ter acomodação adequada e receberem as principais refeições, 43 (53,7\%) e 55 (68,7\%) das mulheres da Maternidade A e B, respectivamente, não usufruíram desse direito, assegurado legalmente.

\section{DISCUSSÃO}

Os dados encontrados apontam que a "lei do acompanhante" não está sendo efetivamente cumprida nas maternidades pesquisadas. As mulheres também não foram orientadas sobre os direitos assegurados por essa lei no pré-natal e durante o internamento, conforme garante a legislação.

Os resultados confirmam que a faixa etária predominante foi de 20 e 35 anos, considerada ideal para a gestação. ${ }^{(8)} \mathrm{A}$ estabilidade da situação conjugal é um fator relevante no desenvolvimento da gravidez, tanto pelo apoio financeiro, como pelo apoio emocional,(9) além de contribuir de forma significativa para uma assistência ao pré-natal adequada, fator verificado em estudos realizados em uma maternidade filantrópica da cidade de São Paulo. ${ }^{(10)}$

Os anos de estudos maior que 8 anos foi constatado em mais da metade das entrevistadas, fator importante na redução da mortalidade perinatal, neonatal e infantil, ligados à escolaridade materna maior.(11) A baixa escolaridade das mulheres, ou seja, menos que cinco anos, é considerada um fator de risco para a gravidez. ${ }^{(12)}$

A maioria das mulheres tinha renda familiar de la 3 salários mínimos, escolaridade média, não brancas e não dispuseram do acompanhante em todo o processo da parturição. Estudo realizado em 2014, estratificado nas cinco macrorregiões do país, identificou que mulheres com rendimentos mais baixos, menor escolaridade, pretas ou pardas, usuárias do SUS, tiveram menos acompanhantes em qualquer momento do parto. $^{(13)}$

Aproximadamente metade das mulheres de ambas as maternidades tiveram participação do acompanhante no pré-natal e 49,3\% confirmaram ser de suma importância essa presença no período gestacional. A presença do acompanhante, especialmente do parceiro, durante o prénatal, em uma maternidade filantrópica da cidade de São Paulo $^{10}$, vem sendo uma experiência produtiva, que contribui satisfatoriamente em todo o processo de nascimento.

Uma grande parte da amostra do estudo desconhecia a Lei 11.108/2005 - "Lei do Acompanhante", corroborando com estudo realizado em maternidade escola de Curitiba, no qual $56,6 \%$ das gestantes desconheciam tal direito(11) e com estudo realizado na Região Sudoeste, com mulheres na fase puerperal que, em sua maioria, desconheciam esse direito, especialmente as primiparas, ratificando as fragilidades nos processos educativos no pré-natal e na atenção ao parto.(14)

Esse desconhecimento vai de encontro a um dos mais violados direitos da saúde que é o direito à informação. A maioria das mulheres não tem sido informada nas consultas de pré-natal e durante a internação sobre a citada lei, apesar da sua vigência há 10 anos. ${ }^{(4)}$ Verifica-se, em geral, que muitas instituições de saúde não se submetem às normas estabelecidas, demonstrando resistência quanto ao direito do acompanhante, subsidiada pelas informações não dadas as gestantes. ${ }^{(15)}$

Constata-se que, mesmo com um percentual pouco expressivo das citações, o enfermeiro foi o profissional que se destacou nas orientações inerentes a informações sobre a referida lei. Por meio de consultas e educação em saúde, em todo o processo gestacional, pré-natal e pós-parto, o enfermeiro está presente, devendo estimular a realização do parto acompanhado, contribuindo para a segurança e autonomia da gestante no processo da parturição. (11) Outro estudo, realizado a partir de consultas pré-natais, ${ }^{(16)}$ revela a importância de sensibilizar os profissionais de saúde sobre a humanização da assistência, durante o trabalho de parto, sendo essencial que esse processo já se inicie no pré-natal, mediante informações e incentivos às gestantes.

Observou-se que menos da metade das mulheres tiveram acompanhante no período do pós-parto imediato, de ambas as maternidades, sendo o companheiro a pessoa mais citada na maternidade $A$, como também identificado na pesquisa Nacional Nascer no Brasil(13). Na maternidade escola de referência no Estado do Ceará(8) foi verificado que a maioria dos acompanhantes no processo de parto, 80,0\%, pertencia ao sexo feminino, sendo mais frequente a mãe da mulher. Para as mulheres da maternidade $B$, foram permitidas somente acompanhantes do sexo feminino, confirmando limitações de livre escolha das parturientes, indo contra o direito legal de ter um acompanhante de livre escolha da mulher, em todas as etapas da parturição.

\section{CONCLUSÃO}

O desrespeito acerca dos direitos das gestantes e parturientes e afalta de informações relativas ao cumprimento 
da "lei do acompanhante," ainda é uma realidade nas maternidades pesquisadas. Tal achado é um convite à reflexão sobre a qualidade e situação das maternidades, segundo os direitos na assistência perinatal, e uma contribuição à política de saúde na área da saúde reprodutiva.

Para a efetividade da Lei do Acompanhante no Brasil, há, portanto, um grande desafio de provocar uma mudança cultural, pautada na adoção do paradigma emergente, com novos valores, atitudes e comportamentos em relação à saúde. Nesse contexto, gestores, profissionais de saúde, pessoas usuárias e o próprio Estado devem disponibilizar condições para que a lei seja cumprida.

É importante que novas discussões e temáticas sejam desenvolvidas, para contribuir de forma significativa, para o cumprimento dessa lei. A educação e treinamento dos profissionais de saúde e uma maior divulgação da "Lei do acompanhante" pela mídia, profissionais de saúde e gestão, têm um papel relevante nesse processo.

\section{REFERÊNCIAS}

1. Barreto MO. O parto na maternidade vinculada: Um direito ainda não alcançado na região nordeste. [Dissertação]. Brasilia (DF): Universidade de Brasilia; 2013. Extraido de [http://repositorio.unb.br/handle/10482/15223] acesso em [20 de abril de 2015]

2. Vargas PB, Vieira BDG, Alves VH, Rodrigues DP, Leão DCMR, Silva LA. A assistência humanizada no trabalho de parto: percepção das adolescentes. Rev.Persqui. Cui. Fundam. (online); 6 (3): 1021-1035, jul/set; 2014.. Extraido de [http://www.seer.unirio.br/index.php/cuidadofundamental/article/ viewFile/3143/pdf_1351], acesso em[19 de Outubro de 2014].

3. Santos RAA, Melo MCP, Cruz DD. Trajetória de humanização do parto no brasil a partir de uma revisão integrativa de literatura. Caderno de Cultura e Ciência, Ano IX, v.13, n.2, Mar; 2015. Universidade Regional do Cariri - URCA Extraido de [http://periodicos.urca.br/ojs/index.php/cadernos/article/ view/838], acesso em [25 abril de 2015].

4. Brasil. Ministério da Saúde. Portaria $n=2.418$, de 02 de dezembro de 2005. Regulamenta, em conformidade com o art. 1 으 da Lei $n=11.108$, de 7 de abril de 2005, a presença de acompanhante para mulheres em trabalho de parto, parto e pós-parto imediato nos hospitais públicos e conveniados com o Sistema Único de Saúde - SUS. Extraido de [http://bvsms.saude.gov. br/bvs/saudelegis/gm/2005/prt2418_02_12_2005.html], acesso em [ 30 de maio de 2015].

5. Brüggemann OM, Ebsen ES, Oliveira ME, Gorayeb MK, Ebele RR. Motivos que levam OS serviços de saúde a não permitirem acompanhante de parto: discursos de enfermeiros. Tex.\& Contexto Enfermagem.2014: 23 (2): 270-7. Extraido de [ http://www.redalyc.org/articulo.oa?id=71431352006 ], acesso em[15 de junho de 2015]

6. Datasus. CnesWeb - Cadastro Nacional de Estabelecimentos de Saúde. Extraido de: [http://cnes.datasus.gov.br/Mod_Ind_Especialidades_Listar aspVTipo $=1128 V$ Listar $=18 V$ Estado $=28 \& V M u n=\& V T e r c=1 \& V S e r v i c o=112 \& \mathrm{~V}$ Classificacao $=005 \& V A m b u=\& V A m b u S U S=\& V H o s p=\& V H o s p S u s=]$, acesso em [17 de fevereiro de 2015]

7. Sergipe. Hospital e Maternidade Santa Isabel.2012. Reunião discute implantação da Rede Cegonha na Maternidade Santa Isabel. Extraido de [ http://hsi-se.com.br/index.php?option=com contentEtask=view\&id=55\&Itemid=1], acesso em [18 de outubro de 2014]

8. Teles LMR, Pitombeira HCS, Oliveira AS, Freitas LV, Moura ERF, Damasceno AKC, et al. Parto com acompanhante e sem acompanhante: a opinião das puérperas. CogitareEnferm. 2010; 15(4):688-94. Extraído de [http://ojs.c3sl. ufpr.br/ojs/index.php/cogitare/article/view/20366/13527], acesso em [10 de julho de 2015].
9. Silva JWF, Silva GBS, Lopes RC. A importância das orientações no pré-natal: o que deve ser trabalhado pelos profissionais e a realidade encontrada. Centro Científico Conhecer.2010; 6(9).. Extraido de [http:// www.conhecer.org.br/enciclop/2010/a\%20importancia.pdf], acesso em [10 de julho de 2015].

10. Corrêa CRH, Bonadio IC, Tsunechiro MA. Avaliação normativa do prénatal em uma maternidade filantrópica de São Paulo. Rev. Esc. Enferm. USP 2011; 45(6):1293-300. Extraido de [http://www.scielo.br/pdf/reeusp/v45n6/ v45n6a03.pdf], acesso em [10 de julho de 2015]

11. Santana MA, Souza SRRK, Gualda DMR, Wall ML. Perfil de gestantes e acompanhantes das oficinas para o parto acompanhado. CogitareEnferm. 2012; 17(1): 106-12. Extraido de [http://www.revenf.bvs.br/scielo. php?script=sci_arttextEpid=S1414-85362012000100015], acesso em[10 de julho de 2015]

12. Brasil. Ministério da Saúde. Pré-natal e Puerpério: atenção qualificada e humanizada - manual técnico. Secretaria de Atenção à Saúde. Brasília. 2006. Extraido de [http://bvsms.saude.gov.br/bvs/publicacoes/manual_ pre_natal_puerperio_3ed.pdf], acesso em[10 de julho de 2015].

13. Diniz CSG, D'Orsi E, Domingues RMSM,Torres JÁ, Bastos Dias MAB, Schneck CA, et al.Implementação da presença de acompanhantes durante a internação para o parto: dados da pesquisa nacional Nascer no Brasil. Cad. Saúde Pública. 2014; 30 (Sup):140-53. Extraido de [http://www. scielosp.org/pdf/csp/v30sl/0102-311X-csp-30-sl-0140.pdf], acesso em [ 30 de maio de 2015].

14. Gottems LBD, Morais TCP, Gonçalves AC, Daniele Ribeiro D, Silva CR, Rodrigues DS. Acesso à rede de atenção a gestação, parto e nascimento na perspectiva das usuárias. Rev. Gestão \& Saúde. 2015; 6(1). Extraído de [http://gestaoesaude.bce.unb.br/index.php/gestaoesaude/article/ view/1141/pdf], acesso em [11 de julho de 2015].

15. Sá RC, Portela IB, Campos ACS. A presença legal do acompanhante na sala de parto. Anais do Gongresso Brasileiro de Enfermagem Neonatal. Fortaleza, Ceará; 2012. Extraido de[http://www.abenfoce.org $\mathrm{br} /$ sites/default/files/A\%2OPRESEN\%C3\%87A\%2OLEGAL\%20DO\%20 ACOMPANHANTE\%2ONA\%20SALA\%2ODE\%2OPARTO.pdf], acesso em [11 de julho de 2015]

16. Santos RAA, MCP Melo, Cruz DD. Trajetória de humanização do parto no brasil a partir de uma revisão integrativa de literatura. Cad. Cult. Ciênc. 2015; 13. Universidade Regional do Cariri. Extraido de http://periodicos. urca.br/ojs/index.php/cadernos/article/view/838/pdf_l], acesso em [10 de julho de 2015]. 